\title{
Towards understanding triiodide photo- chemistry in the solid state by femtosecond electron diffraction
}

\author{
Rui Xian ${ }^{1}$, Stuart A. Hayes ${ }^{1,2}$, Gastón Corthey ${ }^{1}$, Carole A. Morrison ${ }^{3}$, Alexander Marx ${ }^{1,2}$, \\ Valentyn I. Prokhorenko ${ }^{1}$, Cheng Lu ${ }^{4}$, and R. J. Dwayne Miller ${ }^{1,2,4, *}$ \\ ${ }^{1}$ Max Planck Institute for the Structure and Dynamics of Matter, Luruper Chaussee 149, 22761 \\ Hamburg, Germany \\ ${ }^{2}$ Hamburg Centre for Ultrafast Imaging, University of Hamburg, Luruper Chaussee 149, 22761 \\ Hamburg, Germany \\ ${ }^{3}$ School of Chemistry and EaStCHEM Research School, University of Edinburgh, The King's \\ Buildings, West Mains Road, Edinburgh EH9 3JJ, United Kingdom \\ ${ }^{4}$ Departments of Chemistry and Physics, University of Toronto, 80 St. George Street, Toronto M5S \\ 3H6, Canada
}

\begin{abstract}
The photochemistry of the triiodide anion has been investigated by femtosecond electron diffraction. The time-resolved signal indicates the presence of reaction products and large-amplitude coherent motion produced by participating species. To reconstruct the atomic detail of the reaction and identify the major contributors to the detected signal, we outline the approach for atomic-level reconstruction.
\end{abstract}

\section{Introduction}

The triiodide anion is a classic example of small-molecule photochemistry, including photodissociation and geminate recombination. With just three atoms and 3 (bent geometry) or 4 (linear geometry) internal degrees of freedom, it allows a detailed experimental study combined with high-level theoretical insights, but just complex enough to allow the appearance of emergent phenomena such as internal energy transfer. The triiodide anion photochemistry has been studied extensively by transient absorption-type experiments in solution [1-2] and in the gas phase [3]. The solid state equivalent has been much less investigated but creates the possibility of studying coherent inter-molecular effects due to the close proximity and high degree of ordering of the reactive species. However, very little work has been done in the past in this regard, due to the partial reversibility of the reaction as well as the difficulty in preparing sufficiently thin samples with high optical quality for studies in transmission geometries. Previously, one transient absorption study [4] was performed in a single-shot mode at room temperature with an excitation wavelength of $300 \mathrm{~nm}$, while our recent broadband transient absorption study [5] was performed under similar conditions to the electron diffraction work reported here, with $400 \mathrm{~nm}$ excitation and at cryogenic temperatures.

Femtosecond electron diffraction (FED) [6] is a powerful tool for studying such reactions as it provides a direct structural probe of the positions of the atoms in the crystal. However, 
it is not trivial to apply this technique to delicate samples since the number of electrons must be kept low to avoid temporal blurring, resulting in a trade-off between signal-to-noise ratios and time resolution. In addition, it is unclear what the best strategy is for analysis and interpretation of the resulting data in terms of the structural parameters. We therefore use the triiodide system as a test case for developing analysis strategies that will be beneficial to the study of structural changes in photochemical systems.

\section{Experiment}

Crystals of tetra- $n$-butylammonium triiodide $\left(\mathrm{n}-\mathrm{Bu}_{4} \mathrm{NI}_{3}\right)$ were grown from solution evaporation method in ethanol and were microtomed to a thickness of $120 \mathrm{~nm}$ for electron diffraction studies. FED experiments were performed using the apparatus described in [7]. The pump pulses have a center wavelength of $400 \mathrm{~nm}$ and a pulse duration of $\sim 150 \mathrm{fs}$. The electron probe was accelerated to $105 \mathrm{kV}$ with bunch charges of 0.6 and $3.0 \mathrm{fC}$ and a beam diameter of $150 \mu \mathrm{m}$. The repetition rate was reduced to $125 \mathrm{~Hz}$ by mechanical chopping and the sample cooled to $173 \mathrm{~K}$ to allow sufficient sample recovery between laser shots. A variety of time sampling were tested and used for data collection.

High quality electron diffraction patterns were measured and a typical static pattern is shown in Fig. 1. The data was indexed from a series of orientations spanning about 5 degrees around a central orientation used for time-resolved data collection. In some datasets, over two hundred diffraction peaks could be identified, with over one hundred exhibiting statistically significant changes in intensity. The time series data invariably showed a fast initial change in the diffraction peak intensities, with a rise time similar to the instrument response function, and the change persisted for many picoseconds. In addition, a largeamplitude oscillation could clearly be observed with a frequency of $0.6 \mathrm{THz}\left(\sim 20 \mathrm{~cm}^{-1}\right)$. In some instances, there may be faster oscillations present, but they are difficult to distinguish from random noise. The diffraction intensity changes maximize at about 1 ps after photoexcitation and the differential changes are clearly visible by eye (see Fig. 1).
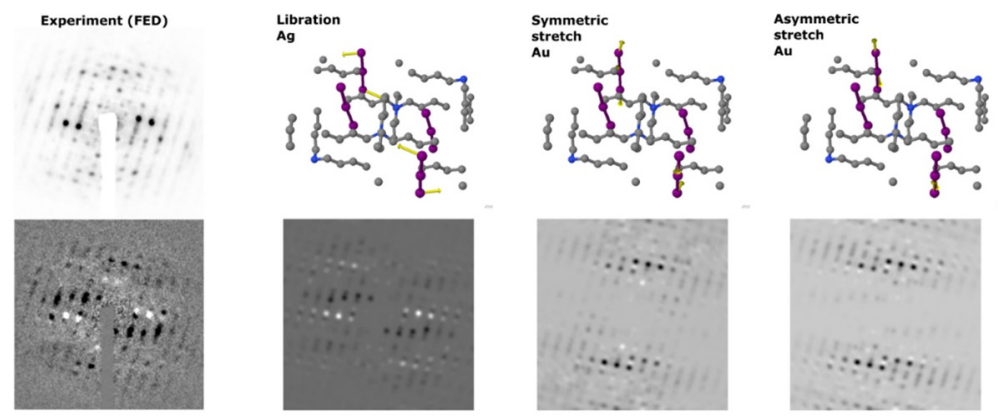

Fig. 1. An electron diffraction pattern of the ground state is shown in the upper left corner, and below it is the differential diffraction pattern recorded at $1 \mathrm{ps}$ after photoexcitation (with light and dark shades being increasing and decreasing intensities, respectively). The next three paired plots to the right show selected ground-state phonon mode displacements and their corresponding effects on the diffraction pattern. The real space pictures on top are viewed from the direction of the electron beam. Each yellow arrow indicates the displacement vector of the corresponding atom. Here, the iodine atoms are depicted in purple, carbon in grey, nitrogen in blue, and hydrogens are omitted for clarity. The resulting changes in the diffraction pattern are presented in the lower three panels. The striking resemblance to the experimental differential diffraction pattern strongly suggests motion of iodine atoms along the libration and symmetric stretching coordinates upon photoexcitation. 


\section{Discussions}

As a first step towards understanding of the FED data, we calculated the displacements associated with the phonon modes of the iodine atoms in the unit cell. We then simulated the overall intensity change in the diffraction pattern corresponding to each phonon mode and used them as a coordinate system to extract the underlying atomic motion from the differential diffraction patterns. This approach yields three types of displacements that agree most strongly with experimental data as shown in Fig. 1.

From this analysis, it appears that the major changes can be described simply as a superposition of the displacements along the three principal modes: the libration, symmetric and asymmetric stretching. Interestingly, the reaction appears to be confined to only one of the two distinct chains in the asymmetric unit of the crystal, since inspection of the simulations due to motion of the other inequivalent triiodide chain in the unit cell gave distinctly different patterns of intensity changes.

\section{Summary}

We have investigated the triiodide anion photochemistry in the solid state using femtosecond electron diffraction. By comparing simulation with experiment, we can associate the diffraction peak intensity changes with atomic displacements along a few principal modes. Further analysis and model testing using a variety of approaches are being investigated to make full use of the data collected in order to gain full understanding of the classic example of reaction dynamics in solid state with atomic-level detail.

This work was funded by the Max Planck Society with additional support from the Hamburg Centre for Ultrafast Imaging. G.C. acknowledges the support from the Alexander von Humboldt Foundation. C.A.M. acknowledge the UK Car-Parrinello Consortium for allocation of computing time on the EPSRC high performance computing resource ARCHER, the EaStCHEM Research Computing Facility and the University of Edinburgh ECDF facility.

\section{References}

1. U. Banin and S. Ruhman, J. Chem. Phys. 98, 4391 (1993)

2. T. Kühne, R. Küster, and P. Vöhringer, Chem. Phys. 233, 161 (1998)

3. R. Nakanishi et al, J. Chem. Phys. 126, 204311 (2007)

4. P. Poulin and K. Nelson, Science 313, 1756 (2006)

5. R. Xian et al, Nat. Chem. 9, 516 (2017)

6. R. J. D. Miller, Annu. Rev. Phys. Chem. 65, 583 (2014)

7. T. Ishikawa, S. A. Hayes et al, Science 350, 1501 (2015) 